Review Article

\title{
PERIODONTAL PROSTHESIS - REVIEW
}

\author{
Vinaya S Bhat ${ }^{1}$, Krishna Prasad D ${ }^{2} \&$ Prakyath Malli ${ }^{3}$ \\ ${ }^{1}$ Professor, ${ }^{2}$ Professor \& HOD, ${ }^{3}$ Post Graduate, Department of Prosthodontics and Crown \& Bridge, A B Shetty \\ Memorial Institute of Dental Sciences, Nitte University, M angalore, Karnataka, India. \\ Correspondence : \\ Prakyath Malli \\ Post Graduate, Department of Prosthodontics and Crown \& Bridge, \\ A.B. Shetty M emorial Institute of Dental Sciences, Nitte University, M angalore - 575 018, Karnataka, India. \\ Mobile : +91 8095727027 E-mail : Prakyath_m@hotmail.com
}

\begin{abstract}
:
Planning a course of treatment for periodontially compromised patients is a multidisciplinary approach involving sequential therapy by a Periodontist and a Prosthodontist. Specific problems have to be recognized and a treatment plan has to be formulated from both the disciplines to deliver best professional and clinical skills in management of a periodontially compromised individual. Hence this review article addresses case characteristics, objectives, sequence of therapy and treatment planning in patients receiving periodontal prosthesis.
\end{abstract}

Keywords: Periodontally involved teeth, Multidisciplinary approach, periodontal prosthesis

Introduction :

Periodontal diseases are a complex condition occurring as a result of etiological factors combined with the systemic condition of the individual. Any individual whose periodontium has been affected will require rehabilitation of both periodontium and affected tooth structure. The incidence of prosthetic treatment in patients with reduced periodontal support is constantly on the rise.

Periodontal prosthetic patient is best managed with joint consultation as a team consisting of Prosthodontist, Periodontist and is frequently to include Orthodontist, Endodontist and Oral Surgeon. Throughout the course of the therapy the team should have a co ordination with each other and patient as well so as each mode of treatment is planned carefully to attain highest success in treatment. ${ }^{1}$

Case characteristics of periodontal prosthetic patient /

Access this article online Quick Response Code

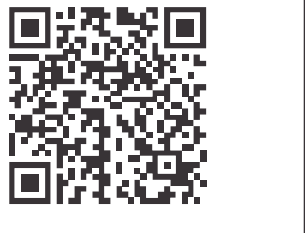

patients requiring periodontal prosthesis

Patients in need of periodontal prosthesis clinically present following situations $^{2,3}$ (Fig $\mathbf{1}$ )

- Periodontitis varying from moderate to advanced stage

- Mobility of the tooth with migration

- Posterior bite collapse

- Mal positioned teeth

- Improperly done restorations contributing to periodontal problems

- Furcation invasions

- Plaque accumulation, root sensitivity, root caries and gingival inflammation due to increased exposure of proximal root concavities and flutings.

- Poor esthetics

- Difficulty in mastication due to absence of stable occlusion

- Para functional habits leading to incisal and occlusal wear

- Radiographicaly - Deep angular infra bony defects, osseous cratering, furcation involvement, horizontal bone loss and loss of lamina dura ${ }^{4}$ (Fig 2)

\section{Objectives of therapy in periodontal prosthetic patients}

The best approach to manage a periodontal prosthetic patient is as a team which consist mainly a Prosthodontist and a Periodontist and sometimes also includes the Orthodontist, Endodontist and Oral surgeon. The team must have co ordination during the course of treatment 
and also communication with the patient is necessary for better treatment outcome. The main purpose of periodontal aspect of the program is to establish a sound foundation in which the final prosthesis will be placed.

Its objectives are , $^{5,6}$

- Removal of local and environmental etiologic factors

- Plaque control and oral hygiene maintenance during the course of therapy

- Removal of pockets

- Restoring osseous and gingival contours

- Removal of furcal invasions by combined periodontal, endodontic and prosthetic procedures.

- Periodic recall and maintenance program.

The goal of the treatment program should establish the physiologic form and function of all the teeth and should also control tooth mobility by mechanical stabilization through splinting. The prosthesis must have the following designs. $^{5,6}$

- It should establish physiologic occlusion

- It should stabilize the mobile teeth

- Development of embrasure form and proximal contact relationships, marginal fit and coronal contours

- Replacing in adequate restorations

- Establishing esthetic and phoneticfeatures.

Following are different types of periodontal prosthesis

1. Hawley Bite Plane Therapy.

2. Provisional Restoration and Stabilization.

3. Periodontal Splints.

4. Restoration Treatment Procedures Using Pins in Periodontal Prosthesis.

5. Telescopic Crown Prosthesis.

6. Semi Precision and Precision Attachment.

7. Gingival Prosthesis.

\section{1) Haw ley bite plane therapy}

The maxillary Hawley appliance with an anterior bite plane is useful in periodontal prosthetic cases in numerous ways. ${ }^{7}$
Whenever there is occlusal trauma this appliance serves the following purpose

- Provides rest for neuro musculature and periodontal attachment apparatus

- Posterior occlusal disarticulation

- Control of local etiologic factors causing inflammation

- Enhance healing of occluso traumatic lesions. ${ }^{8,9}$

\section{2) Provisional restoration and stabilization (fig 3 )}

Provisional restorations help in management of periodontal prosthetic patient and are placed during the surgical phase of therapy. One of the most important functions of provisional restoration is stabilization of mobile teeth. However, during the early phases of initial therapy will result in decrease in tooth mobility through the control of inflammation and initial occlusal adjustments, lesions of irreversible and progressive tooth mobility must be treated. ${ }^{10,11}$

\section{3) Periodontal splints}

Splinting is mechanical joining to teeth to enhance their ability to withstand forces placed on them. Splinting of teeth whether temporary or permanent, is adjunctive therapy; it will not cure periodontal disease. However dental splinting can prevent

a) Pathologic migration

b) Retain teeth in position

c) Facilitate treatment during scaling, curettage, and periodontal surgery

d) Support teeth so that the effect of occlusal interferences can be more readily located and removed by occlusal equilibration

e) Stabilize teeth so that occlusal forces are distributed to a combination of teeth rather than to individual teeth.

\section{Indications and contraindications of splinting Indications}

a) Usually, FPD's and splints are preferable to RPD's with advanced loss of periodontal support. They provide rigidity and a more favorable force distribution to the remaining periodontium. ${ }^{12}$ 
b) There is an agreement that splinting of mobile teeth and FPD's, after periodontal and initial occlusal therapy is indicated when mobility is increasing and interfering with chewing ability and comfort. Lindhe indicates splinting for

- Progressive mobility of the tooth as a result of gradual increasing width of the periodontal ligament in teeth with reduced height of the alveolar bone.

- Increased bridge mobility despite splinting

c) Progressive mobility can be controlled by uni lateral splints even though resistance to bucco or labiolingual mobility is less than mesiodistal mobility.

d) Increased bridge mobility requires cross arch splinting. The main objective of splinting is to produce an environment where total mobility of the splint is normal or at least no longer increasing. ${ }^{13}$

e) Nyman et al. have demonstrated long term splint stability despite minimal periodontal support and hyper mobility of isolated abutment teeth. ${ }^{14}$

\section{Contraindications}

a) Most patients with moderate periodontitis, having slight or no detectable mobility after periodontal treatment, do not require fixed splints

b) Lindhe described two situations

1. Increased mobility of the tooth with increased width of the periodontal ligament, but normal height of the alveolar bone

2. Increased mobility of a tooth with increased width of the periodontal ligament and reduced height of the alveolar bone. ${ }^{12}$

c) Splinting is contraindicated in patients with gingivitis. Resolution of inflammation by root planning and reduction of occlusal prematurities will usually significantly reduce detectable mobility. 12,15,16

d) Patients with advanced periodontitis have varying numbers of teeth that exhibit severe bone loss, advanced mobility, and edentulous areas.

\section{Classification of stabilization by Splinting}

Teeth can be splinting by several methods.

A) Temporarystabilization
I. Removable extra coronal splints
II. Fixed extra coronal splints
III. Intracoronal splints
IV. Etched metal resin bonded splints.

B) Provisional stabilization
I. Acrylic splints
II. M etal bond and acrylic splints.

C) Longterm stabilization
I. Removable splints
II. Fixed splints
III. Combination removable and fixed splints

\section{4) Restorative treatment procedures using pins in periodontal prosthesis (Fig 4 )}

Parallel and Non parallel pin techniques are used in dentistry for teeth that exhibit mobility. It is temporarily splinted for cases exhibiting marked mobility to re evaluate prognosis, if the teeth is firm then prognosis is more favorable after which permanent stabilization is instituted.

\section{Advantages of pin retained restoration}

1. Retention of restoration

2. Conservation of tooth structure

3. Operatorschair time isless

4. Good esthetics of natural labial and buccal enamel

5. Hygiene better than complete veneer crowns

6. Reduced laboratory procedures

7. Less cost than complete veneer crowns

8. Good stabilization of mobile teeth can be achieved by parallel pin technique

\section{Disadvantages of parallel pin technique}

1. Possibility of encroachment upon dental pulp

2. More attention to detail needed by the operator

3. Technicians un familiar with the technique

4. Abutment restoration might become loose if depth of the pins into sound tooth structure is not sufficient

5. If buccal and lingual walls are thin and un supported enamel fracture might occur

\section{Advantages of non parallel pin technique}

These advantages are in addition to those of parallel pin technique 
1. May be used with crowded or maltose teeth

2. No necessity of paralleling device. ${ }^{17}$

5) Telescopic crown prosthesis in severe periodontal destruction (Fig 5 )

Telescopic crowns prosthesis consists of covering the prepared teeth with thin gold copings, or thimbles, that in corporate shoulder on the gingival margins to which super structure is abutted with temporary luting agents. ${ }^{18}$ The surface of the copings are un polished because cement will not adhere to a polished surface. The copings may be soldered or removed as individual units with the superstructure that provides splinting. Often these two approaches are integrated in a single case. If complete arch splinting is necessary, cross linkage of abutments across soldered copings allows a bilateral splint to be divided into manageable units. ${ }^{19}$

\section{Advantages:}

1. It is difficult to prepare the walls of the short teeth to a minimal taper for resistance form. Copings are contoured to nearly parallel axis to overcome this problem.

2. Paralleling of severely tipped abutment teeth is possible without orthodontics.

3. Full arch periodontal splinting is accomplished in multiple smaller segments.

4. Abutment teeth are protected if superstructure is dislodged.

5. Super structurestemporarily cemented can be removed for treatment of recurrent periodontal disease.

6. Teeth with minimal prognosis can be intentionally included in a splint, which can be eventually extracted with the abutment crown converted into poetic

\section{Disadvantages:}

1. Retention between the coping and tooth must be greater than between super structure and coping

2. Fit is difficult between the super structure margins and coping finish lines

3. Esthetic limitations in the anterior areas due to the bulk of the double castings with narrow embrasure spaces

4. In short abutment teeth with narrow embrasure spaces telescoping is contra indicated.

\section{6) Semi precision attachment and precision attachment in terminal borderline cases (Fig 6 )}

Whenever there is a periodontally involved arch principles of stabilization and intra arch support can be achieved with intra coronal semi precision or precision attachment. As a general rule removable partial dentures with extra coronal clasps should not be used as a splinting vehicle as they create forces too great for periodontally involved teeth to withstand. The intra coronal retainers on the other hand, allow for better control of bucco lingual, mesio distal and vertical forces.

\section{Advantages of intra coronal retainers over extra coronal clasping}

1. It prevents lateral stresses in the periodontium of the retainers during insertion or removal, and stabilizes the abutment teeth against lateral forces. With intra coronal retainer's attachments, the forces of function are directed vertically.

2. The intra coronal attachments use parallel walls rather than undercuts to resist the forces of displacement.

3. It can be used for short clinical crowns, and it eliminates the display of clasps. ${ }^{20}$

\section{Disadvantages}

1. Intra coronal attachments require prepared abutments and castings; but in the case of periodontally involved and mobile teeth, splinting is usually recommended.

2. They also require complicated clinical and laboratory procedures.

3. Eventually wear, with resultant loss of frictional resistance to denture removal

4. They are difficult to repair or replace

5. Intra coronal attachments are effective in proportion to length, thus they are least effective in short teeth. It is true that at least two third of the length of the manufactured precision attachment must be used for satisfactory results, but now with the milled in semi precision cases and other developed techniques, this is no longer a factor of concern. 


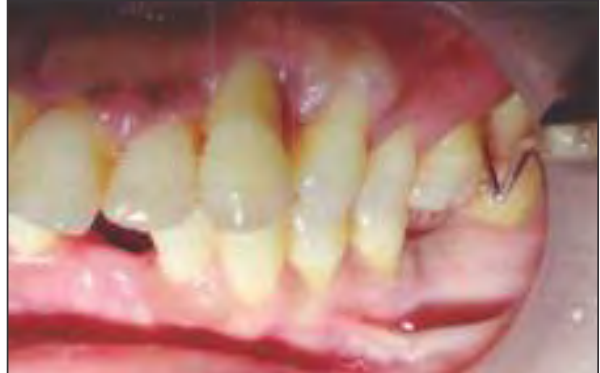

Figl. : Example of periodontal prosthetic patient

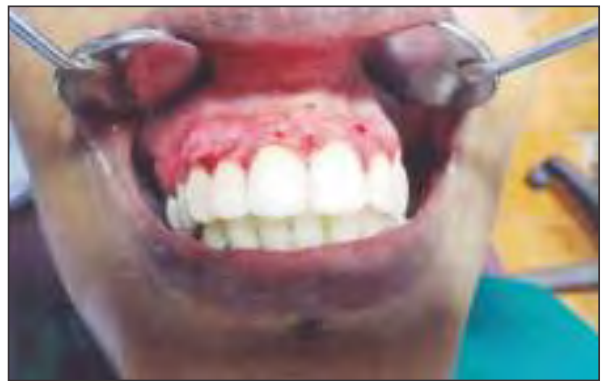

Fig3. : Provisional restoration used as a splint to stabilize mobile teeth

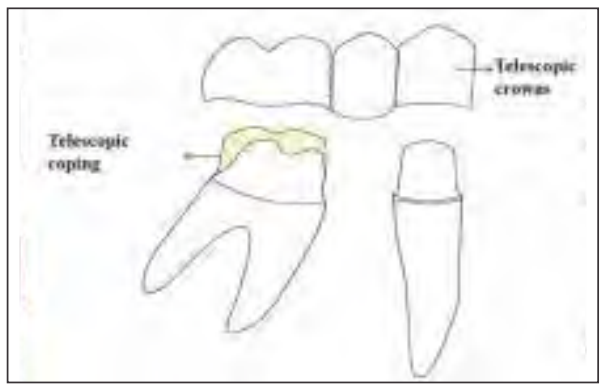

Fig 5. : Telescopic crown prosthesis

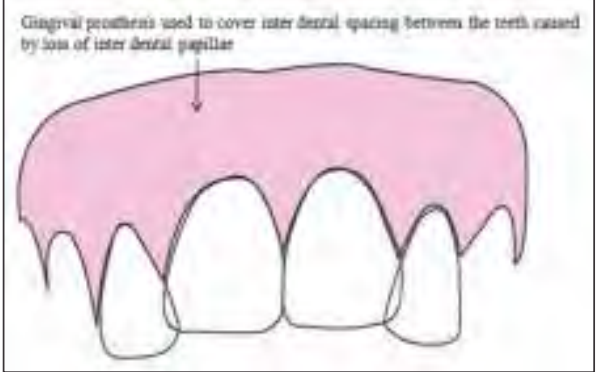

Fig7. : Gingival prosthesis

\section{7) Gingival prosthesis (Fig 7 and 8 )}

Gingival replacement prostheses have historically been used to replace lost tissue when other methods of regenerative procedures are considered un predictable or impossible, with this method, large tissue volumes are easily replaced.

Tissue replacement prostheses are used to replace tissue lost through surgical gingival procedures, trauma, ridge

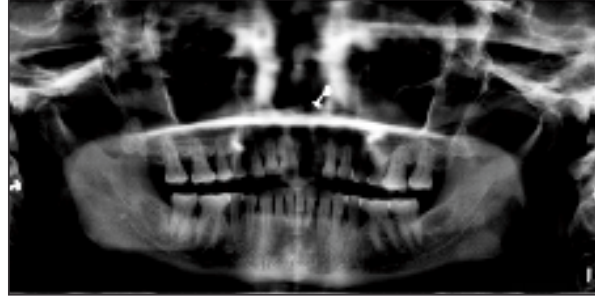

Fig2. : Radiographic assessment

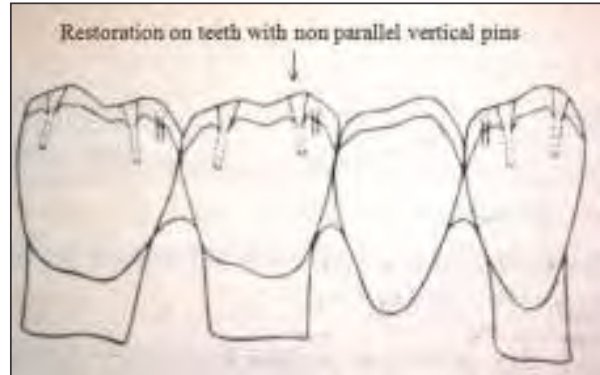

Fig4. Periodontal splint positioned on the teeth

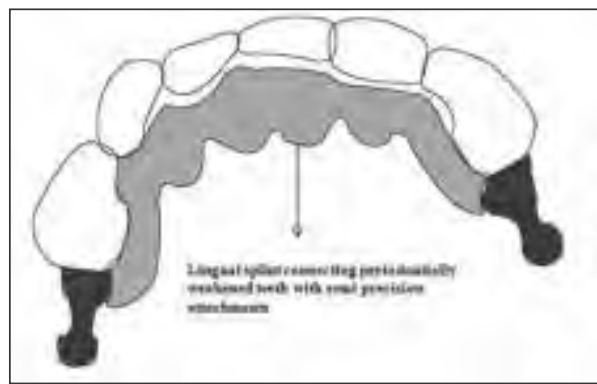

Fig6. : Semi Precision attachment

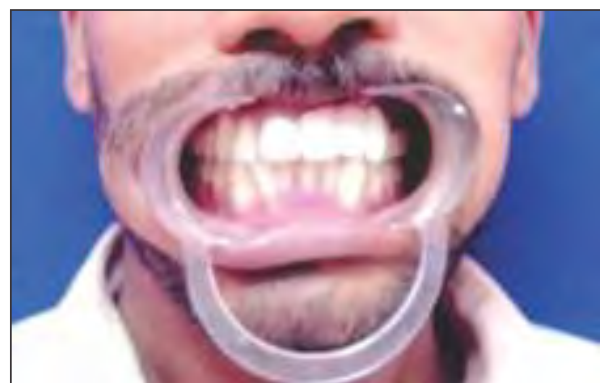

Fig8. : Gingival prosthesis used to cover ridge defects

resorption or traumatic tooth extraction. Materials used for gingival prostheses include pink auto cure and heatcured acrylics, porcelains, composite resins and thermoplastic acrylics, as well as silicone-based soft materials.

Gingival defects are treated with surgical or prosthetic approaches. Minor procedures to rebuild papillae and 
grafting procedure s that may involve not only soft-tissue manipulation but also bone augmentation to support the soft tissue. With the success of these treatments the result mimics the original soft tissue contours. It is possible to create esthetically pleasing and anatomically correct tissue contours when small volumes of tissue are being reconstructed, but this method is unpredictable when a large volume of tissue is missing. The surgical costs, healing time, discomfort and unpredictability make this choice unpopular.

Prosthetic replacement, with acrylics, composite resins, porcelains and silicones, is a more predictable approach to replacing lost tissue architecture. It is especially useful when a larger amount of tissue needs replacement. Ideal tissue contours can be waxed, processed and then colored to match the surrounding tissue. The patient need not undergo any additional surgical procedures and receives an esthetically pleasing, functional restoration. It is possible to show the patient a waxed-up result or even take a try in prosthesis directly to the mouth for evaluation before significant treatment is initiated. ${ }^{21}$

\section{Discussion :}

Prosthodontic treatment of a patient with periodontally

\section{References:}

1. Meyers H.E, Baraff L.S. A restorative approach to periodontally treated mouth. Dent clin north am 1965; 9: 13.

2. Amsterdam M. Periodontal prosthesis. In: Goldman H, Cohen DE (eds). Periodontal therapy, ed 5. St louis: mosby, 1973:990.

3. Stahl S.S: Marginal lesion. In Goldman H.M, Cohen D.W (eds): Periodontal therapy, Ed 4. St. Louis: mosby 1968; 120-121.

4. Rosenberg M. Periodontal and prosthetic management for advanced cases: quintessence publication co Inc, 1988.

5. Meyers H.E, Baraff L.S. A restorative approach to periodontally treated mouth. Dent clin north am 1965; 9: 13.

6. Rosenberg M.M. Management of osseous defects, furcation involvement and periodontal pulpal lesions. In clark jw (ed) : Clinical dentistry, vol 3, chap 10. Hagerstown, md: Harper and row, 1979.

7. Heckert L. Prerestorative therapy using a modified hawley splint. J prosthet dent 1980; 43:126.

8. Amsterdam M. Periodontal prosthesis. Twenty five years in retrospect. Alpha omegan, 1974; 67: 29-31.

9. M iller G.M , Kreuzer D.W. The modified hawley appliance. part II. Int j periodont rest dent 1982; 2(1): 29-45.

10. Amsterdam M , Fox L: Provisonal splinting: Principles and techniques. Dent clin north am1959; 3: 73-99.

11. Lindhe J, Nyman S. The role of occlusion in periodontal disease and the biological rationale for splinting in treatment of periodontitis. Oral sci rev1977; 10:11. involved teeth is a multidisciplinary approach. It includes fabrication of a simple fixed prosthesis to complicated procedures like full mouth rehabilitation. Each patients presenting with different clinical situations has challenging treatment to be formulated by the operators. Sequence of therapy, combination of different aspects of treatment and operator skill play an extremely critical role in the success of managing such patients. The objectives of treating these patients have been arrived at removal of all etiologic factors, treating the cause of periodontal breakdown, prosthetic stabilization and maintaining oral health with recall program. The array of prosthesis includes bite plane appliances, splints to precision attachment and telescopic crowns. Gingival prosthesis is another form of periodontal prosthesis given for replacing lost soft tissues. Accurate diagnosis holds a key for the longevity of treatment success in these cases.

Patient cooperation is yet another aspect for good management of patients. Since it is a multidisciplinary approach, several appointments may be required to complete the course of treatment and patient compliance in maintaining also plays a major role in long term success. This review article has compiled all aspects of treatment with periodontal prosthesis.

12. Lindhe J. Textbook of clinical periodontology. Copenhagen. 1983, Munksgaard; 466-479.

13. Lindhe J, Nyman S. The role of occlusion in periodontal disease and the biological rationale for splinting in treatment of periodontitis. Oral sci rev1977; 10:11.

14. Nyman S, Lind he J, Lundgren D. The role of occlusion for stability of fixed bridges in patients with reduced periodontal support. J Clan Periodontol1979; 50: 163-169.

15. Keel W, Sisisky H, Phillips C. The effect of splinting on tooth mobility. I. During initial therapy.J Clan Periodontal 1979; 6:45-58.

16. Polson A.M , M eitner S.W , Sander H.A. Reversibility of bone loss due to trauma alone and trauma super imposed upon periodontitis. Periodont 1976; 11:290.

17. Gredier A, William R. Periodontal prosthesis. The c.v. M osby company st Louis; 1968.

18. Pichard J.F, fedrer,m. A modern adaptation of the telescopic principle in periodontal prosthesis.J periodontal 1962; 33:360-364.

19. Pugh C.E, Smerke J.W. Rationale for fixed prosthesis in the management of advanced periodontal disease. Dent Clin North Am 1969; 13(1): 243-262.

20. Goodman J, Goodman H.W: Balance of force in precision free end restorations.J Pros Dent 1963;:13:302.

21. Barizlay I, and Tamblyn I: Gingival prosthesis- review. J Can Dent Assoc $2003 ; 69(2): 74-8$. 